\title{
Système d'information et de modélisation de la vulnérabilité pastorale pour la gestion et la prévention des crises au Sahel
}

\author{
Erwann Fillol ${ }^{1 *}$ Frédéric Ham $^{2}$ Alex Orenstein ${ }^{1}$
}

\section{Mots-clés}

Bétail, pastoralisme, biomasse, pâturages, ressource en eau, système d'alerte rapide, vulnérabilité, Sahel

Accepted: 9 April 2020

Published: 23 September 2020

DOI: $10.19182 /$ remvt.31903

\section{Résumé}

L'élevage pastoral au Sahel est extrêmement dépendant des irrégularités climatiques, et particulièrement de la qualité de la saison des pluies qui conditionne la repousse des pâturages et le remplissage des points d'eau. Les éleveurs nomades et transhumants adaptent leurs stratégies de déplacement à l'état et à la disponibilité de ces ressources. Les outils de suivi de la sécurité alimentaire de ces territoires pastoraux doivent donc intégrer, en plus des données spatialisées sur la biomasse, des informations sur ces mouvements pastoraux et sur les facteurs qui les conditionnent. Pour accompagner les systèmes d'alerte locaux au Sahel, I'organisation non gouvernementale Action contre la Faim propose un modèle de vulnérabilité pastorale basé sur des données satellitaires acquises en temps réel et sur la connaissance des pratiques pastorales. Ce modèle permet d'alimenter en informations un système d'alerte précoce en identifiant les zones d'intérêt pastoral déficitaires en ressources plusieurs mois avant la période de soudure potentiellement problématique. La confrontation avec I'information concomitante collectée sur le terrain par des relais sentinelles permet une surveillance de la situation pastorale, contribuant au développement des infrastructures ou au déploiement de l'aide humanitaire.

- Comment citer cet article : Fillol E., Ham F., Orenstein A., 2020. Information and modeling system of pastoral vulnerability for crisis management and prevention in the Sahel. Rev. Elev. Med. Vet. Pays Trop., 73 (3): 169-177, doi: 10.19182/remvt.31903

\section{INTRODUCTION}

Les systèmes d'alerte précoce (SAP) mis en place par les pays sahéliens à la suite d'années de grandes sécheresses constituent encore aujourd'hui la composante principale en matière d'information d'alerte pour mieux adapter les systèmes d'intervention d'urgence pour la sécurité alimentaire et nutritionnelle des ménages vulnérables. Ils permettent de définir des actions d'appui à l'amélioration des conditions de vie des populations affectées par les variations climatiques, les chocs pluviométriques, et les crises alimentaires et nutritionnelles. Ils assurent ainsi le lien entre l'alerte précoce et la planification des réponses et s'intègrent dans les cadres régionaux de

1. Action contre la Faim, Dakar, Sénégal.

2. Médecins sans Frontières, Genève, Suisse.

* Auteur pour la correspondance

Email : erwann.fillo1@gmail.com concertation incluant les autorités sous-régionales politiques et techniques, les agences de l'Organisation des Nations unies et les organisations non gouvernementales comme le Réseau de prévention des crises alimentaires au Sahel et en Afrique de l'Ouest (RPCA) ou le Dispositif régional de prévention et de gestion des crises alimentaires (PREGEC). Enfin, à partir d'un outil d'analyse de la vulnérabilité des populations, le cadre harmonisé $(\mathrm{CH})$, élaboré en commun et validé par de nombreuses institutions (CILSS, 2018), ils produisent un consensus des bilans de situation.

Dans les pays sahéliens et aux échelles locales, les cadres institutionnels et les contextes agroenvironnementaux et socioéconomiques ont certes évolué avec un développement de la pluriactivité, mais le disponible vivrier dans les zones agricoles représente encore aujourd'hui le champ d'investigation nécessitant le plus de développement. Cela pose la question, d'une part, de l'amélioration de la collecte de données chez les populations relevant de différents systèmes d'activité (agriculture, élevage, etc.) et pour divers critères d'environnement et de mode de vie (santé, nutrition, etc.), et, d'autre part, de l'intégration de ces données pour instruire et accompagner les décisions politiques. 
Dans le cadre d'une approche projet, l'organisation non gouvernementale Action contre la Faim (ACF) s'appuie sur ces constats et s'intègre dans les réflexions et les dynamiques actuelles des institutions nationales et sous-régionales. Elle prend en compte les avancées, les contraintes, les besoins et les stratégies existants sur ces dispositifs de SAP.

L'amélioration des dispositifs peut se faire par différentes voies : l'analyse intégrée de la sécurité alimentaire et nutritionnelle ; le développement de réseaux pour la mise en place de collaborations entre les structures ; le renforcement des relations entre les différents niveaux d'information (sous-régional, national et local), le renforcement de l'anticipation de l'alerte ; le repositionnement géographique des instruments de réponse et l'amélioration du ciblage socioéconomique des bénéficiaires ; et la collecte des données de terrain et satellitaires.

Cette logique demande notamment une coordination renforcée et une participation des différents acteurs concernés (institutions gouvernementales, collectivités territoriales, société civile, instituts de recherche, autres organisations non gouvernementales, entre autres). Les dispositifs régionaux existants tels le Comité permanent interEtats de lutte contre la sécheresse dans le Sahel (CILSS) et le Réseau de systèmes d'alerte précoce sur la famine (FEWSNET) suggèrent des analyses de la vulnérabilité des communautés par l'emploi d'indicateurs englobant les milieux agricoles et pastoraux, mais sans la prise en compte spécifique des caractéristiques inhérentes au pastoralisme, c'est-à-dire la complémentarité agroécologique des espaces, l'accessibilité aux ressources et la mobilité des éleveurs. En effet, l'usage d'un indice de végétation permet une caractérisation spatiale de la production de biomasse d'une année par rapport à une normale, mais ne permet pas la prise en compte des autres aspects du pastoralisme et de l'élevage nomade et pastoral. Au-delà de l'intégration d'indicateurs de suivi de variables physiques (pâturages et eau), la considération de la vulnérabilité pastorale implique de compléter l'analyse notamment en y intégrant le facteur de mobilité.

En effet, la mobilité « permet aux pasteurs de tirer avantage des ressources qui sont d'une grande variabilité dans le temps et dans l'espace » (Marty et al., 2006). Parmi les différentes formes de mobilité, la transhumance correspond à des mouvements saisonniers qui permettent de « valoriser les complémentarités interzonales, sur des espaces souvent très vastes » et qui, « loin d'être une simple habitude, correspond[ent] en fait à une véritable construction sociale, politique, économique, sans cesse renouvelée et réadaptée » (Bonnet et al., 2005).

Dès lors, la considération de la mobilité oblige à dépasser l'approche classique d'évaluation des ressources pastorales existantes, pour aborder la problématique de l'accessibilité à ces ressources dans l'espace et dans le temps et tenter de relever le défi de l'établissement de bilans fourragers des espaces pastoraux.

La considération des mouvements et stratégies spatiales d'adaptation des éleveurs vise à compléter les analyses de vulnérabilité pastorale habituellement focalisées sur les indicateurs biophysiques. Les outils régionaux de surveillance et d'alerte précoce (CILSS, FEWSNET) n'autorisent pas jusqu'à présent une finesse d'analyse correspondant aux contraintes sociales rencontrées, liées, entre autres, à l'usage de l'espace pastoral, aux considérations culturelles et aux aspects sécuritaires.

Le développement du modèle de vulnérabilité pastorale est motivé par l'ambition d'aller au-delà des considérations physiques, partant du principe que le pastoralisme sahélien est non seulement dépendant de la répartition et de l'abondance des ressources, mais également des usages que les éleveurs font de l'espace. Il s'agit donc d'intégrer dans l'analyse le mode de vie pastoral, c'est-à-dire l'ensemble de ces « stratégies qui constituent à la fois un système de production et un mode de vie éprouvé, adapté aux aléas et risques du contexte sahélosoudanien » (Ancey et Monas, 2005).
D'autres initiatives se sont intéressées à la surveillance du pastoralisme au Sahel et à la mise en place de systèmes d'information. Parmi elles, citons le Système d'information pastoralisme et environnement au Sahel (SIPES) de la FAO (PESAH, 2004), basé sur un réseau de la société pastorale et de décideurs politiques dans le but de promouvoir une gestion durable des ressources pastorales (Toutain et al., 2010), en intégrant une composante environnementale qui met en évidence la relation réciproque entre les systèmes d'élevage et les écosystèmes. Partant des acquis du SIPES, le Système d'information sur le pastoralisme au Sahel (SIPSA) a été élaboré pour suivre les dynamiques pastorales au Sahel (Ickowicz et al., 2005), permettant l'élaboration d'un atlas des systèmes pastoraux au Sahel et de leurs évolutions (Touré et al., 2012).

Tout en s'inscrivant dans cette continuité, les outils de gestion de surveillance des ressources pastorales sur la zone sahélienne élaborés par ACF considèrent avant tout la problématique écologique (comment régénérer les écosystèmes ?) et alimentaire (comment alimenter les troupeaux ?), et tentent de porter des éléments de réponse à la question prédominante de prévention des conflits et de l'aménagement du territoire (Marty, 2000). La plus-value de ces outils est de rendre l'information et les données facilement visualisables et publiquement accessibles par l'utilisation d'une plateforme de visualisation cartographique interactive avec une mise à jour de l'information en temps réel.

\section{MATERIEL ET METHODES}

\section{Contexte}

Sur le terrain, les actions mises en œuvre dans le cadre du renforcement des dispositifs SAP concernent le suivi de la vulnérabilité pastorale pour la définition de réponses appropriées à ces groupes de population dans la sous-région ouest-africaine. ACF, en partenariat avec les ministères de l'élevage du Mali, du Niger et du Burkina Faso, et les associations d'éleveurs au Sénégal, a développé un système d'alerte précoce pastorale utilisant des données de télédétection satellitaire, des enquêtes de terrain, en tenant compte des connaissances, des pratiques locales et des traditions (Orenstein, 2017a ; Ham et Fillol, 2012 ; Ham et al., 2011 ; Bastide et al., 2008 ; Fillol et al., 2008 ; Métais, 2007). Des indicateurs adaptés issus de la télédétection spatiale, utilisant des traitements pour extraire l'information statistique et le ciblage géographique, ont été élaborés en vue d'appuyer la prise de décision à une échelle d'analyse régionale permettant de considérer de larges espaces et ayant l'avantage de donner une visualisation synthétique de la vulnérabilité à l'échelle sous-régionale (Ham et Fillol, 2012).

Les principaux outils développés concernent a) la quantification et le suivi des ressources pastorales à partir de données de télédétection (production de biomasse végétale et disponibilité en eau de surface), et b) le suivi des mouvements et des stratégies d'adaptation des éleveurs et des troupeaux au gré des saisons. Les produits issus de ces outils permettent d'alimenter en informations le modèle de vulnérabilité pastorale.

\section{Suivi de la production de biomasse}

Le BioGenerator (Fillol, 2013), outil développé et utilisé par ACF depuis 2007, permet de mesurer la production de biomasse annuelle en établissant le cumul sur la saison de croissance des valeurs de productivité quotidienne données par les productivités de matière sèche (DMP) (Jacobs et al., 2006). Le produit DMP décadaire est obtenu à partir des acquisitions de la série de capteurs satellitaires SPOTVEGETATION entre 1998 et 2013, succédés par PROBA-V depuis 2014, et distribué par le programme de l'Union européenne Copernicus Global Land Service (CGLS). Le résultat est la production totale 
de biomasse, pour chaque saison depuis 1998, exprimée en rendement à l'hectare (kilogrammes de matière sèche végétale produite par hectare) et avec une résolution spatiale d'un kilomètre. La valeur cumulée obtenue à la fin de la période de croissance donne la quantité de biomasse annuelle et permet de dresser des cartes de production de biomasse et d'anomalie de production de biomasse (par rapport à la moyenne calculée sur l'ensemble des années depuis 1998) sur l'étendue du Sahel.

Les données en sortie de BioGenerator ont fait l'objet de plusieurs travaux de validation, intégrant des données in situ de mesure de biomasse sur le Mali, le Sénégal et le Niger et sur plusieurs années (Fillol, 2013). A la différence des indices de végétation comme l'indice de végétation par différence normalisée (NDVI), habituellement employés comme indicateur de l'abondance de pâturage, le BioGenerator permet de disposer de mesures quantitatives et relatives de production de biomasse, sans nécessiter des travaux additionnels de calibration à partir de mesures in situ qui impliqueraient l'organisation régulière, sur une base annuelle, de missions de terrain. En utilisant un système d'information géographique (SIG), il est possible d'agréger et de représenter spatialement les quantités de biomasse végétale produites selon les unités géographiques d'analyse choisies (par exemple découpages administratifs, unités agroécologiques, zones d'intérêt pastoral) permettant de réaliser des bilans zonaux de production (Ham, 2013).

\section{Suivi de la disponibilité en eau de surface}

La méthode est basée sur l'utilisation du produit des petites étendues d'eau (small water bodies, SWB) (Bartholomé et Combal, 2006) accessible à une fréquence temporelle décadaire, à une résolution spatiale d'un kilomètre, et informant de manière booléenne de la présence ou de l'absence d'eau de surface. Le produit SWB, issu du même programme CGLS, provient d'acquisitions de la même série de capteurs satellitaires SPOT-VEGETATION (1998-2013) et PROBA$\mathrm{V}$ (depuis 2014) et a fait l'objet de travaux de validation terrain ; il donne des résultats bons en commission (92,8 à 98,7\%), nettement moins bons en omission, ceci étant dû notamment à la faible résolution du produit (Haas, 2010 ; 2009).

En intégrant cette information ponctuelle de présence d'eau, l'HydroGenerator (Fillol, 2010), outil développé par ACF en 2010, produit des cartes donnant une information statistique d'un indice d'accessibilité à l'eau de surface pour les éleveurs. Le calcul de l'indice se fait par l'intégration du temps de remplissage de tous les points d'eau présents dans un rayon d'action défini, pondéré par la distance aux points d'eau.

L'étape suivante envisagée est de coupler cette information concernant l'eau de surface avec un inventaire exhaustif des points d'eau fonctionnels, puits et forages, afin de mieux estimer la disponibilité en eau et d'en déduire une accessibilité différentielle des pâturages présents. Cependant, cet inventaire est aujourd'hui partiel à l'échelle des pays du Sahel et ne peut prétendre refléter la situation réelle et exhaustive de la couverture en points d'eau. Le modèle de vulnérabilité intègre donc pour le moment seulement le suivi des points d'eau de surface détectés par satellite. Ainsi, l'accessibilité des pâturages au-delà du rayon d'influence des mares n'est pas réduite à zéro mais maintenue à un niveau minimum afin de pallier la non-prise en compte des points d'eau fixes ainsi que les omissions de détection des points d'eau de surface causées par la faible résolution des SWB.

\section{Indice de vulnérabilité lié à la biomasse}

L'indice de vulnérabilité est créé pour pallier la lacune de l'analyse simple par anomalie qui, en comparant l'année en cours avec l'ensemble des années antérieures, est insensible aux enchaînements d'épisodes secs. Si les éleveurs peuvent surmonter un épisode sec isolé, ils sont beaucoup plus sensibles à une récurrence de sécheresses consécutives pouvant avoir des effets destructifs sur les troupeaux (Fafchamps et Gavian, 1998). Le principe de l'indicateur de vulnérabilité lié à la biomasse VI est basé sur un calcul récursif décrit par les équations 1 et 2 : pour chaque année, la contribution au calcul de

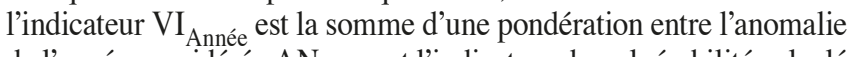
de l'année considérée $\mathrm{AN}_{\text {Année }}$ et l'indicateur de vulnérabilité calculé pour l'année antérieure $\mathrm{VI}_{\text {Année-1 }}$ suivant une pondération donnée par le facteur $\alpha_{\mathrm{VI}}$ fixé à $50 \%$ :

$$
\mathrm{VI}_{\text {Année }}=\alpha_{\mathrm{VI}} \times \mathrm{AN}_{\text {Année }}+\left(1-\alpha_{\mathrm{VI}}\right) \times \mathrm{VI}_{\text {Année-1 }}
$$

avec

$$
\mathrm{AN}_{\text {Année }}=\frac{\text { Biomasse }_{\text {Année }}}{\frac{\sum_{\mathrm{A}=\text { Année }}^{1998}\left(1-\alpha_{\mathrm{VI}}\right)^{(\text {Année-A }) \times \text { Biomasse }_{A}}}{\sum_{\mathrm{A}=\text { Année }}^{1998}\left(1-\alpha_{\mathrm{VI}}\right)^{(\text {Année-A })}}}-1
$$

où $\mathrm{VI}_{1998}=0$ et $\alpha_{\mathrm{VI}}=0,5$

\section{Enquêtes hebdomadaires des conditions pastorales}

L'imagerie satellitaire seule n'est pas suffisante pour établir le suivi en temps réel de l'ensemble des conditions pastorales. Si le BioGenerator donne l'information de production de végétation et la quantité de biomasse produite durant la saison de croissance, son utilisation ne permet pas de quantifier la biomasse encore présente ni sa qualité durant la période sèche. En outre, de nombreux autres éléments influencent la vulnérabilité des communautés pastorales et sont indétectables par satellite, parmi eux l'état de fonctionnement des puits, les conditions de circulation et d'accès aux pâturages, les conditions de marché, et l'état de santé des animaux.

L'enquête de terrain classique dans les zones pastorales est coûteuse et chronophage, nécessitant des ressources importantes pour couvrir de vastes territoires souvent difficilement accessibles. Le ciblage des communautés pastorales présente un véritable défi logistique et méthodologique. Pour pallier ces contraintes, une méthode de suivi des zones pastorales à distance a été développée utilisant des enquêtes téléphoniques via le système de messages courts téléphoniques (SMS). Dans chaque pays où est établi le suivi (aujourd'hui Mali, Niger, Burkina Faso et Sénégal), des sites sentinelles de surveillance pastorale sont sélectionnés dans les villages se trouvant dans les zones de concentration pérennes ou saisonnières, et sur les axes de transhumance habituels. Les sites d'intérêt ainsi que les indicateurs sont identifiés en concertation entre les ministères de l'élevage, les organisations pastorales et ACF. Pour chaque site, un « point focal» sélectionné, le plus souvent un vétérinaire, un agent du ministère de l'élevage ou un représentant d'une organisation d'élevage, reste en contact régulier avec les pasteurs de la zone. Cette personne est chargée d'envoyer les informations collectées de manière hebdomadaire par SMS en répondant à un questionnaire préétabli. Les messages reçus sont centralisés, puis reliés à une base des données géospatiales dont l'information résultante est synthétisée et cartographiée de manière bimestrielle.

Les informations collectées par le réseau de sites sentinelles concernent principalement la disponibilité en pâturage et en eau, la concentration de bétail, le suivi des mouvements habituels ou inhabituels, les prix de marché, la présence d'épizooties, l'embonpoint et l'état de santé des animaux, et la présence de feux de brousse. Les indicateurs sont produits de manière qualitative, par l'appréciation du point focal en réponse au questionnaire. Ce système permet une collecte rapide de l'information tout en s'appuyant sur les savoirs traditionnels et l'implication des communautés pastorales. 


\section{Cartographie des mouvements pastoraux}

La connaissance de la localisation des couloirs et des mouvements de transhumance ainsi que des zones de concentration est indispensable pour appréhender la vulnérabilité pastorale au Sahel, car elle permet d'isoler et d'étudier les zones d'importance pastorale sur les cartes de disponibilité de biomasse et d'accessibilité à l'eau. Ces informations sont collectées à l'occasion d'exercices de cartographie participative basés sur une méthodologie d'évaluation, par les éleveurs eux-mêmes, de la vulnérabilité pastorale et de la capacité de charge des terroirs (ACF, 2013). L'objectif est de cartographier les ressources de la communauté, les risques et les dangers, et la réponse donnée par les stratégies d'adaptation. Ces ateliers participatifs résultent de l'adaptation de la boîte à outils d'ACF de cartographie communautaire au contexte pastoral se focalisant sur les mouvements saisonniers des éleveurs.

Des fonds de cartes produits au niveau régional (découpage administratif de niveau 1) sont utilisés durant les ateliers participatifs. Des représentants d'organisations pastorales, des vétérinaires, des techniciens de la reproduction animale ainsi que des délégués des ministères de l'élevage se réunissent et s'accordent sur la définition des paramètres et sur la méthodologie de mesures comme la saisonnalité, les zones de transit et de concentration, les mouvements normaux, et les mouvements de contingence ou alternatifs. Les participants des ateliers dressent les cartes au niveau régional qui sont ensuite validées au niveau national par les membres des organisations pastorales et les ministères de l'élevage. Ce travail continu de cartographie a débuté en 2011 au Mali, au Niger, en Mauritanie et au Sénégal, et une mise à jour a été effectuée en 2016.

L'information issue des cartes finalisées est numérisée et superposée aux cartes de production de biomasse et d'accessibilité à l'eau de surface provenant de la télédétection satellitaire. La superposition de l'information du stock de biomasse et d'eau de surface produite en fin de saison des pluies avec les routes connues de transhumance permet une prédiction de la capacité de charge durant la saison sèche et l'identification des zones potentiellement à risque. Par exemple en cas d'anomalies négatives de production de biomasse et de présence d'eau de surface sur une zone de concentration importante en saison sèche, les éleveurs nomades seront probablement contraints d'éviter le secteur, tandis que les éleveurs sédentaires risquent de subir une détérioration de leurs conditions. La figure 1 montre les principaux mouvements de retour de transhumance en début de saison d'hivernage rapportés par le Réseau Billital Maroobè, association de regroupement d'éleveurs au Sahel et partenaire d'ACF.

\section{Modèle de vulnérabilité pastorale}

Le modèle de vulnérabilité pastorale est une construction méthodologique, en cours de développement, destinée à évaluer la vulnérabilité des communautés pastorales face aux épisodes de sécheresse. Le modèle vise également à estimer l'évolution de cette vulnérabilité dans le temps et l'espace. Sans toutefois prendre en compte de manière exhaustive l'ensemble des dimensions socioéconomiques et des moyens de subsistance des agropasteurs, l'intégration par le modèle des composantes fondamentales du pastoralisme se base sur deux postulats : a) les troupeaux constituent l'élément fondamental des moyens d'existence des éleveurs pasteurs, et b) la subsistance du cheptel est directement dépendante de son approvisionnement en pâturages et de son abreuvement en eau.

Les sorties du modèle sont des produits cartographiques permettant d'appuyer l'identification des zones où l'activité pastorale est potentiellement exposée à un déficit en ressources et d'évaluer le niveau de risque découlant de cette pénurie. Les cartes produites permettent l'analyse et l'évaluation de la situation pastorale, et d'anticiper les comportements et les mouvements des éleveurs durant la saison sèche à venir. La figure 2 discrétise les différents niveaux d'analyse du modèle détaillés ci-après.

Le niveau 1 d'analyse permet de déterminer la quantité de biomasse végétale totale produite par unité de surface ou rendement. Il est globalement atteint par l'utilisation du produit DMP. Il établit une connaissance de la situation générale concernant la végétation d'une zone pastorale mais sans distinction de la qualité de la biomasse (herbacée, ligneuse ou agricole). C'est à ce niveau d'utilisation directe des valeurs de DMP que l'indice VI de vulnérabilité lié à la biomasse est calculé.

Le niveau 2 vise une quantification de la production de la biomasse végétale appétible communément dénommée pâturage. La biomasse appétible est une fraction de la biomasse totale dont le ratio est fonction des espèces en présence mais aussi du contexte de pénurie, car la sélectivité des animaux diminue en période de crise. La littérature donne un facteur de conversion normal évoluant de $30 \%$ en climat soudanien (sud de l'isohyète $600 \mathrm{~mm}$ ) à $50 \%$ en climat sahélien (nord de l'isohyète 400 mm) (Toutain et Lhoste, 1978).

Le niveau 3 vise l'estimation de la quantité produite de pâturage accessible en fonction de la topographie et de la distance aux points d'eau. En fonction du paysage, de l'espèce et de la taille du troupeau, le déplacement maximal quotidien observé se situe aux alentours de 20 kilomètres (Milleville, 1992) mais cette distance peut, dans certaines conditions et contraintes, être supérieure (Ouedraogo, 1994).

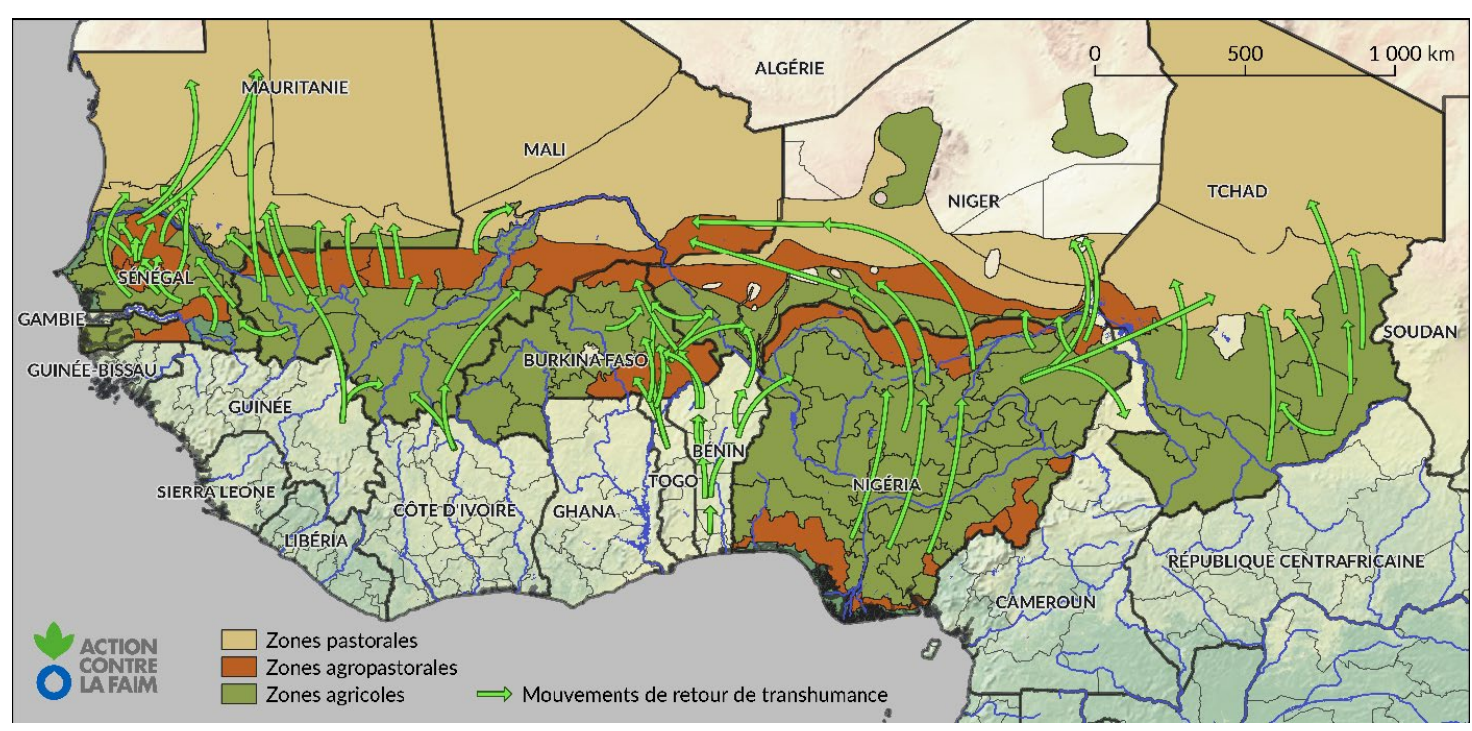

Figure 1 : mouvements de retour de transhumance en début d'hivernage 2018 au Sahel. 


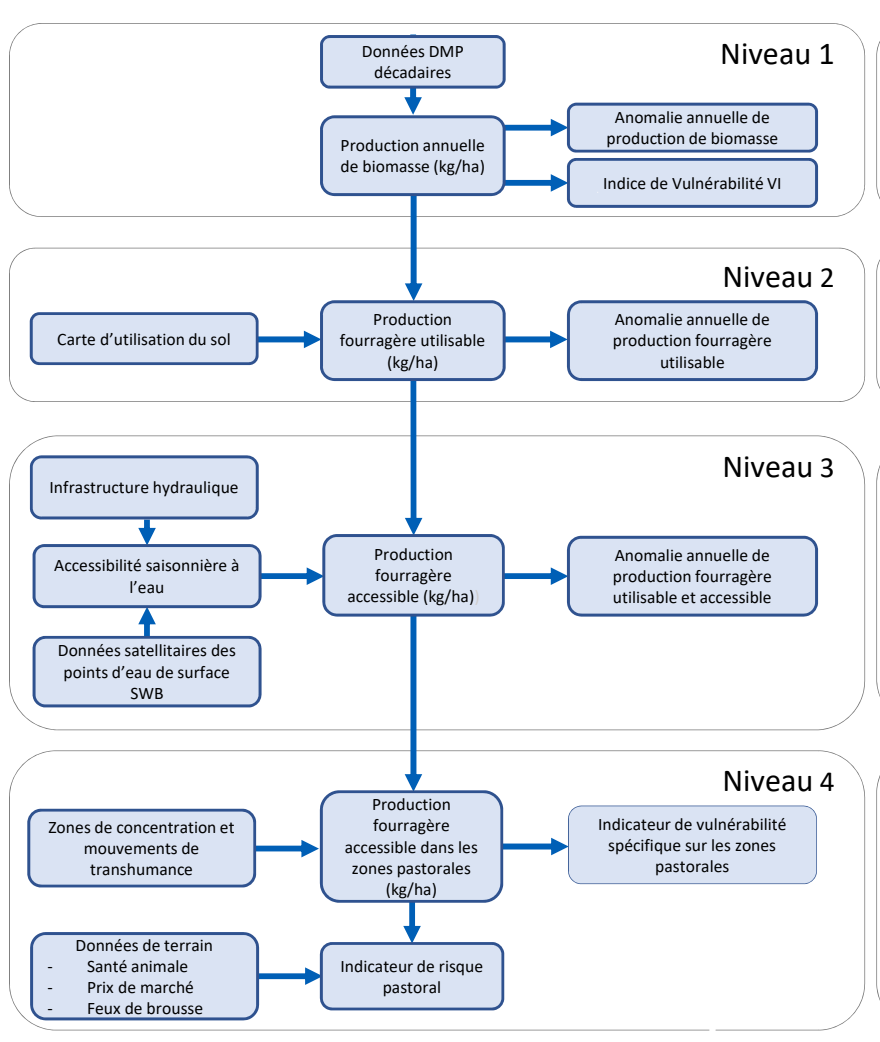

En se basant sur ces observations, l'aire d'influence des mares, des puits et des forages est fixée empiriquement à 30 kilomètres qui est la distance considérée par le système de parcours quotidien maximal des éleveurs entre un point d'eau et une zone de pâturage. L'accessibilité aux pâturages est pondérée de façon graduelle suivant une courbe gaussienne entre $100 \%$ au voisinage immédiat du point d'eau et $30 \%$ à la distance de 30 kilomètres et au-delà. Idéalement, les contraintes extérieures comme les conflits ou les contraintes spatiales (frontières, obstacles naturels, etc.) devraient pouvoir être intégrées afin de refléter une situation particulière ou exceptionnelle.

Le niveau 4 ajoute la connaissance de terrain. En croisant l'information de production de biomasse et d'accessibilité à l'eau avec la délimitation des zones de concentration et la localisation des couloirs de transhumance, il devient possible d'identifier les zones présentant une dégradation de la situation pastorale. Les informations collectées sur ces zones via le réseau des sites sentinelles permettent d'identifier des risques additionnels pour la communauté pastorale.

\section{RESULTATS}

Aujourd'hui, les résultats principaux du système d'alerte précoce mis en place par ACF et adopté par les services techniques des SAP sont basés sur l'analyse des indicateurs suivants :

- l'évaluation de la biomasse disponible. Le calcul de l'anomalie annuelle de production de biomasse est la comparaison de l'année en cours par rapport à l'ensemble des années couvertes par les données satellitaires, c'est-à-dire depuis 1998. C'est ce premier niveau de résultats qu'utilisent les SAP du Mali et du Niger et leurs partenaires pour effectuer le ciblage géographique des zones à risque. La figure 3 montre la carte de l'anomalie de production de biomasse pour la saison d'hivernage 2018 issue du BioGenerator début octobre 2018;

- l'évaluation de la vulnérabilité aux sécheresses consécutives. Le calcul récursif de l'anomalie (équations 1 et 2) permet d'analyser l'année en cours avec la succession des années antérieures proches. Le résultat permet d'identifier les zones de sécheresses récurrentes,

- Identification des
zones vulnérables aux
sécheresses
Identification des
zones de production
de biomasse
anormalement faible

Figure 2 : différents niveaux d'analyse de la donnée de biomasse végétale au Sahel. $D M P$ : productivités de la matière sèche ; SWB : petites étendues d'eau phénomène particulièrement critique pour le pastoralisme. C'est ce niveau d'analyse qui est actuellement pris en compte par le $\mathrm{CH}$. La figure 4 montre la carte de l'indice de vulnérabilité de la biomasse VI pour l'année 2018 issue du BioGenerator début octobre 2018 sur lequel sont superposées les zones habituelles de concentration en saison sèche et froide ;

- le suivi de l'accessibilité à l'eau de surface. Le calcul de l'anomalie de présence d'eau pondérée par la distance aux points d'eau permet une analyse statistique cartographique de l'accessibilité à l'eau. Ce suivi peut contribuer au ciblage géographique des zones vulnérables en caractérisant l'accessibilité aux pâturages. La figure 5 montre la carte de l'anomalie de l'accessibilité à l'eau de surface sur le Sénégal pour la période juin-juillet 2018 issue de l'HydroGenerator début août 2018 ;

- la localisation des parcours et des mouvements des pasteurs et des troupeaux, ainsi que des zones de pâturage en relation avec les périodes et les stratégies d'utilisation (zones de concentration en saison sèche, en saison des pluies, ou en saison froide, zones de replis ou de concentration). Des travaux en ce sens ont été réalisés par ACF au Mali, au Niger et partiellement en Mauritanie afin de produire une première série de données déjà validées à l'occasion d'ateliers nationaux au Mali et au Niger, auxquels ont participé des membres des directions de l'élevage, des instituts de recherche, des ONG et des associations d'éleveurs. La figure 6 montre l'exemple sur le Sénégal des informations de concentration et de mouvements de troupeaux observées par les relais sentinelles pastoraux sur la période juin-juillet 2018 ;

- l'informations de terrain sur les conditions pastorales. Les données de terrain, collectées par le réseau de sites sentinelles à travers le projet de surveillance pastorale d'ACF, sont utilisées pour la création de bulletins bimestriels concernant le Mali, le Niger, le Burkina Faso et le Sénégal. Ces bulletins informent les autorités et les acteurs humanitaires sur les conditions pastorales actuelles. Le modèle de collecte de données par téléphone portable / SMS permet une flexibilité et un déploiement pour d'autres utilisations de surveillance concernant l'eau et les conditions sanitaires et de santé par exemple. La figure 7 

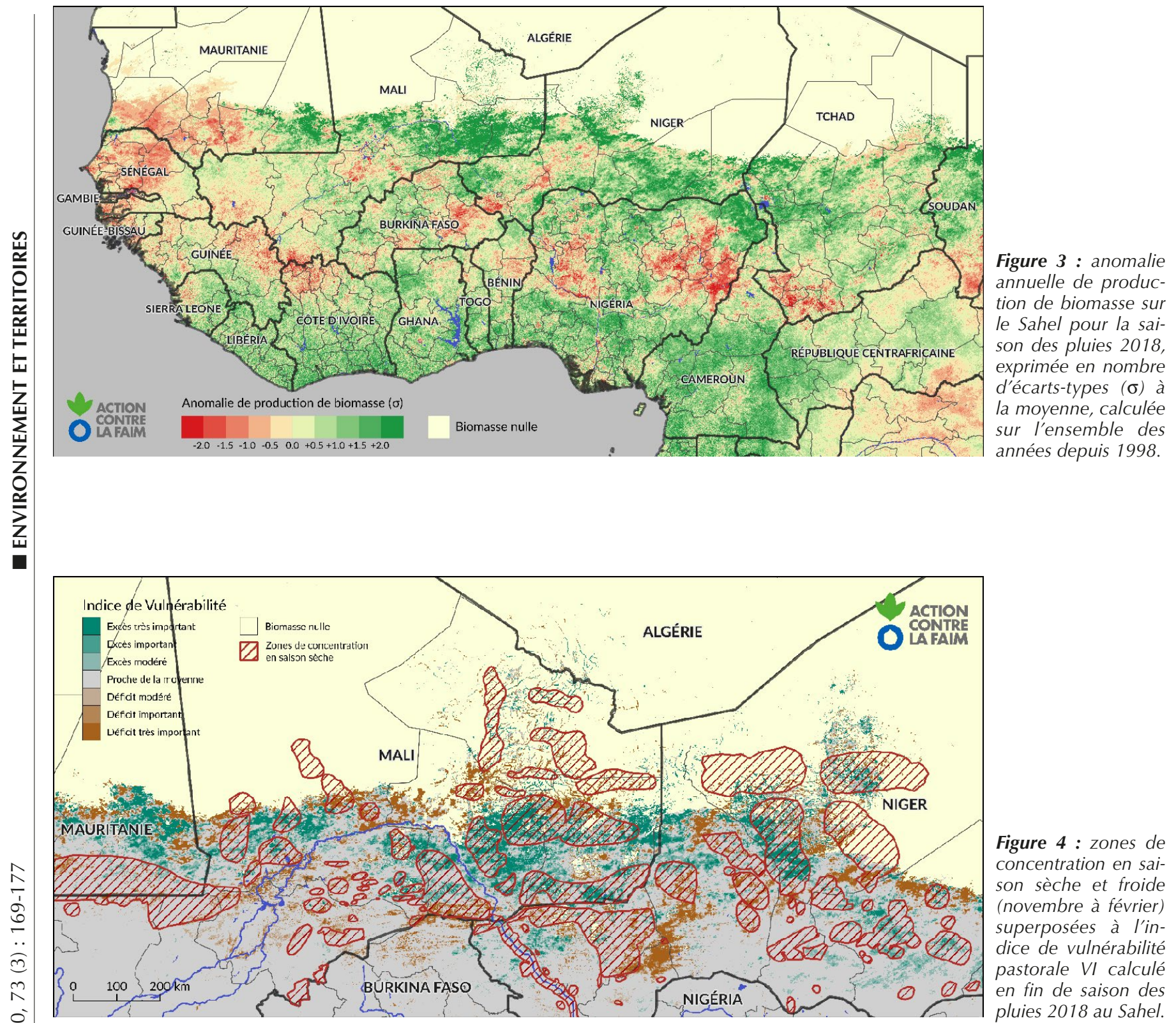

Figure 4 : zones de concentration en saison sèche et froide (novembre à février) superposées à l'indice de vulnérabilité pastorale VI calculé en fin de saison des pluies 2018 au Sahel.

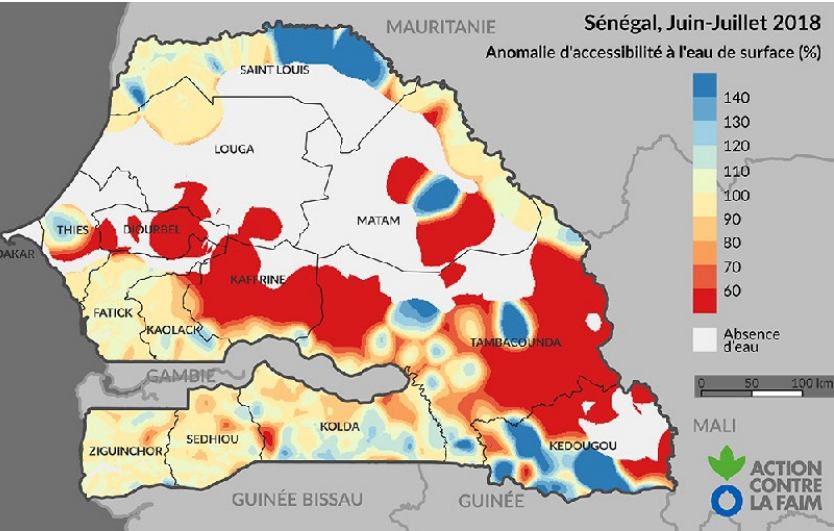

Figure 5 : anomalie d'accessibilité à l'eau de surface en juin-juillet 2018 sur le Sénégal par rapport à la situation normale pour la même période sur l'ensemble des années depuis 1998.

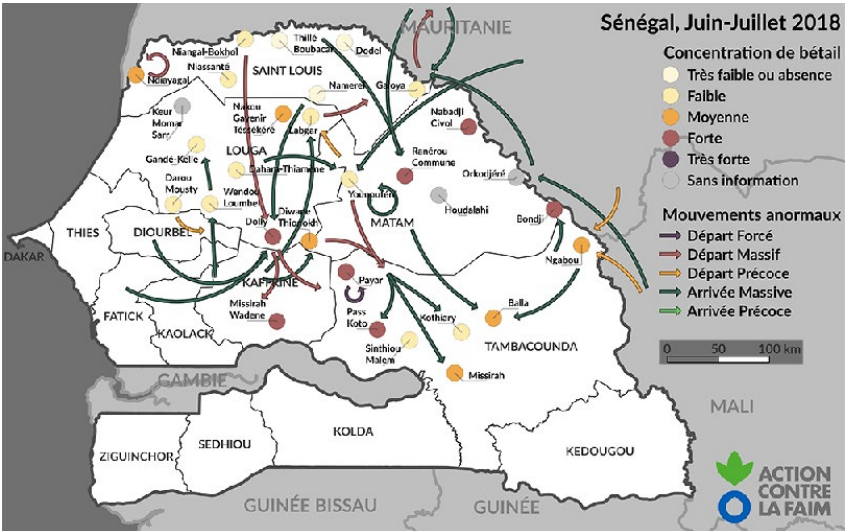

Figure 6 : concentration du bétail et mouvements anormaux rapportés par les relais sentinelles pastoraux en juin-juillet 2018 au Sénégal. Départ Forcé : en raison de l'insécurité, de conflits ou de maladies animales ; Départ Massif : en nombre inhabituel ; Départ Précoce : en raison du manque de ressources ; Arrivée Massive en nombre inhabituel; Arrivée Précoce : avant la date normale. 


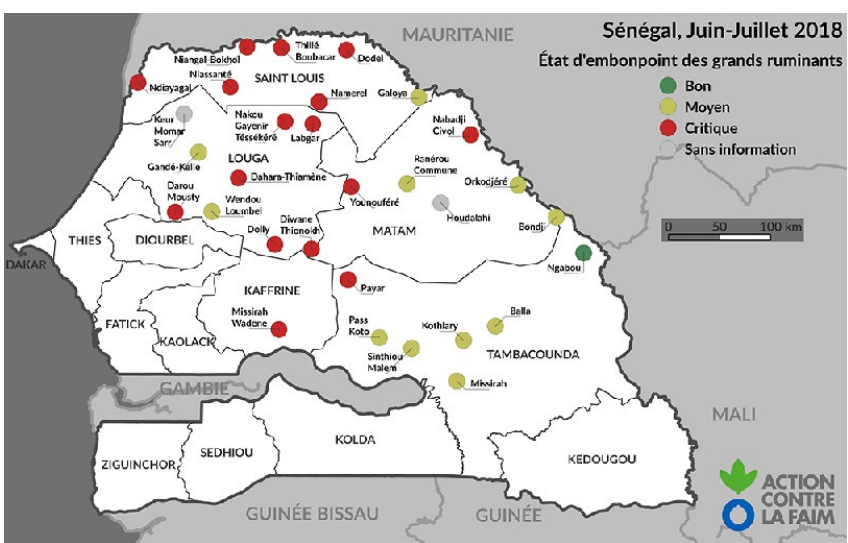

Figure 7 : état d'embonpoint des grands ruminants rapporté par les relais sentinelles pastoraux en juin-juillet 2018 au Sénégal.

montre l'exemple au Sénégal de l'information d'état d'embonpoint des animaux relevée en juin-juillet 2018.

L'ensemble de ces indicateurs permet d'évaluer la vulnérabilité des populations des zones pastorales et agropastorales face à l'insécurité alimentaire et nutritionnelle. Afin d'aboutir à un ciblage des communautés à risque, cette évaluation de la vulnérabilité est réalisée par un opérateur expert à partir de l'analyse visuelle de plusieurs couches d'information géographique. Dès la fin de la saison des pluies, il est en effet possible d'identifier les zones déficitaires en ressources (pâturage et eau de surface), d'anticiper l'évolution probable de l'accessibilité à ces ressources et de présumer les zones abandonnées ainsi que les zones de concentration propices aux risques de tensions intercommunautaires, de propagation de maladie et de mortalité animale. Les zones déjà identifiées comme « zones à risques alimentaires » ou présentant déjà une prévalence de malnutrition élevée les années antérieures peuvent être ciblées prioritairement pour un suivi spécifique.

\section{DISCUSSION}

Les résultats de cette méthode, aujourd'hui toujours en cours de développement, sont déjà appréciés par les acteurs de la sécurité alimentaire (les autorités politiques et techniques, les agences des Nations unies et les ONG). ACF produit annuellement depuis 2010 les cartes d'anomalie de production de biomasse en fin de saison des pluies, soit avec six mois d'avance sur la saison sèche à venir et la période de soudure potentiellement problématique. Elles sont intégrées comme source de données pour les SAP des pays du Sahel et sont utilisées au Niger, au Mali et au Sénégal lors des analyses du CH, ou pour établir les profils d'« analyse de l'économie des ménages » afin d'évaluer le niveau d'insécurité alimentaire des populations pastorales (Paridaens, 2017).

Les outils employés ont permis de renforcer l'alerte précoce au Sahel, particulièrement pour l'anticipation et la caractérisation des crises (Ford, 2013 ; Roucaute, 2012 ; Fillol et al., 2008), et aussi pour l'identification des zones de sécheresse ou de soudure précoce (Rouse, 2018 ; Peyton, 2017 ; FAO-PAM, 2017 ; FSNWG, 2017). Par exemple lors de la crise alimentaire de 2017-2018 au Sahel, ACF, par l'utilisation de ses outils, a été dès le mois d'août 2017 le premier acteur capable de diffuser une alerte sur la situation pastorale à la communauté humanitaire (FSNWG, 2017 ; Orenstein, 2017b ; RPCA, 2017 ; RFI, 2017). Les outils ont aussi permis d'identifier les zones à risque pour l'élaboration du plan de réponse apporté par le Bureau de la coordination des affaires humanitaires (OCHA) (OCHA, 2018).
La fourniture d'éléments d'analyse dès la fin de la saison des pluies en octobre permet de bénéficier d'une fenêtre d'intervention de plusieurs mois dans les zones identifiées comme vulnérables par le modèle. La large gamme des données intégrées dans le modèle permet de considérer plusieurs types de risques parmi lesquels : la pénurie de pâturages, le manque d'eau de surface, les mouvements de transhumance anormaux, la surconcentration de troupeaux et l'apparition d'épizooties. La collecte de données de terrain concernant les prix des marchés et l'état de santé et d'embonpoint des animaux complète l'analyse.

Les outils d'ACF sont utilisés systématiquement par les SAP nationaux du Mali et du Burkina Faso pour effectuer leurs propres analyses de biomasse (SAP Mali, 2017 ; PRAPS-BF, 2017). Le CH de suivi de la vulnérabilité des populations au Sahel mis en place par le CILSS, dont la fonction est d'établir une synthèse des différentes sources de données sur la situation alimentaire dans le Sahel, intègre l'analyse de la situation pastorale produite par ACF à travers les réunions des comités techniques nationaux et régionaux (Paridaens, 2017 ; CILSS, 2018).

La mise en ligne du portail d'information SigSahel (www.sigsahel. info), donnant un lien d'accès à la plateforme de cartographie interactive GeoSahel, rend accessibles publiquement les résultats de ce travail. Les données et rapports peuvent y être téléchargés librement, pour être rediffusés et réutilisés. De plus, la combinaison avec d'autres bases de données humanitaires concernant la nutrition et la sécurité alimentaire permet d'exploiter encore davantage la capacité d'alerte précoce du système.

Les efforts à fournir demeurent toutefois importants, en particulier concernant l'opérationnalité des outils, mais ouvrent également de nouvelles perspectives dans le cadre de ce processus de construction, parmi elles : a) l'extension du transfert de ces outils à d'autres pays sahéliens comme la Mauritanie et le Tchad ; b) l'amélioration de la fiabilité et de la finesse, et l'automatisation du calcul des indicateurs dans les systèmes de suivi des pays aboutissant à l'établissement de bilans fourragers réalistes ; c) la diffusion de l'information et l'appropriation du système par les communautés pastorales ; d) le repositionnement des stratégies et des instruments proposés dans les plans de réponse aux différents niveaux en termes de prévention et de gestion des risques pour le pastoralisme ; et e) la combinaison avec un modèle de prédiction de rendement ou de suivi de production agricole pour un système d'alerte précoce plus complet des risques d'insécurité alimentaire des zones agropastorales.

Concernant le point c, un exemple de diffusion de l'information est le projet pilote d'Adaptation des technologies durables pour les pasteurs maliens (STAMP), mis en œuvre en 2015, qui utilise les sorties du système pour alimenter un centre d'appel téléphonique au Nord Mali. Les agents utilisent une interface accessible par internet (www. stamp-mali.org) donnant accès aux données concernant la quantité de biomasse et fournissent aux pasteurs l'information du niveau de croissance de la végétation dans les zones d'intérêts. La collecte de données de terrain et la cartographie participative ont été utilisées afin de compléter l'information donnée par le centre d'appel directement aux éleveurs (prix de marché, niveau de concentration en bétail).

Pour le point $\mathrm{d}$, il s'agit principalement de renforcer les articulations du modèle de vulnérabilité pastorale avec les interventions et les projets des partenaires humanitaires ; le développement repose notamment sur des approches de « réduction des risques de catastrophe » et de protection sociale (filets de sécurité alimentaire et nutritionnelle) dans les pays concernés.

Le système de surveillance pastorale devrait encourager la mise en œuvre d'initiatives pilotes d'alerte précoce communautaire et ainsi participer au renforcement de la résilience des populations pastorales 
au niveau local (communes, communautés et ménages). Par exemple au Niger l'Enquête participative de vulnérabilité et de capacités, outil d'analyse multisectorielle développé par ACF en partenariat avec le département de géographie de l'Université Abdou-Moumouni à Niamey et l'Association pour la redynamisation de l'élevage au Niger, a permis de mettre en évidence les facteurs de vulnérabilité des ménages pastoraux et les moyens mis en œuvre pour l'adaptation aux urgences (Illiassou, 2017).

L'objectif est une meilleure compréhension des systèmes d'élevage par la mise en place de travaux d'étude et de recherche autour des caractéristiques socioéconomiques et environnementales, en considérant de nouveaux indicateurs pouvant être intégrés dans le système de suivi de la vulnérabilité : effectifs de cheptel, étude des termes de l'échange et des prix de marché, indicateur de vulnérabilité au niveau des ménages, mobilité différentielle.

\section{CONCLUSION}

L'approche développée par ACF pour son système de surveillance pastoral a permis d'atteindre des résultats probants pour l'amélioration des outils d'analyse de la vulnérabilité des populations pastorales face à l'insécurité alimentaire et nutritionnelle au Sahel. Elle a lancé les bases d'une initiative située à la confluence de la géographie physique et sociale pour une gestion améliorée des risques de catastrophe et aussi pour une réflexion portant sur l'avenir des systèmes d'élevage sahéliens, leur évolution et les stratégies d'adaptation liées aux effets du changement climatique. ACF et ses partenaires techniques et institutionnels envisagent de poursuivre ce travail afin d'intégrer au mieux cette initiative dans leur volonté de contribuer durablement à une augmentation de la résilience des communautés pastorales sahéliennes face aux catastrophes naturelles et ainsi lutter contre la malnutrition.

\section{Remerciements}

Les auteurs remercient l'ensemble des contributeurs financiers et bailleurs de projets qui ont subventionné la mise en place du système, principalement : la Banque mondiale, la Fondation Albert II de Monaco, le Fonds de recherche d'ACF, International Fond Policy Research Institute (IFPRI), UN Food and Agriculture Organization (FAO), et Office of US Foreign Disaster Assistance (OFDA).

\section{Déclaration des contributions des auteurs}

$\mathrm{EF}, \mathrm{FH}$ et $\mathrm{AO}$ ont participé à la gestion du projet de surveillance pastorale d'ACF en Afrique de l'Ouest, à la mise en place et au développement du système, à la collecte des données, à la rédaction, et à la révision de cet article.

\section{Conflits d'intérêts}

L'étude a été réalisée sans aucun conflit d'intérêts.

\section{REFERENCES}

ACF, 2013. Action contre la Faim, Etude Participative des Risques, Vulnérabilités et Capacités Communautaires Manuel Pratique, 138 p., www. actioncontrelafaim.org/wp-content/uploads/2018/01/acf_2013_-_manuel_ pratique_epvc.pdf

Ancey V., Monas G., 2005. Le pastoralisme au Sénégal, entre politique " moderne » et gestion des risques par les pasteurs, In: Tiers-Monde. La question alimentaire en Afrique : Risque et politisation (sous la direction de Janin P. et de Suremain C.E.), 46 (184) : 761-783, doi: 10.3406/ tiers.2005.5610

Bartholomé E., Combal B., 2006. Small Water Bodies. In: VGT4Africa User Manual (first ed), Office for Official Publication of the European Communities, Luxembourg, $271 \mathrm{p}$.
Bastide J., Fillol E., Métais T., 2008. Evaluation des risques liés aux variations spatiotemporelles de la pluviométrie au Sahel. 13th IWRA World Water Congress, Montpellier, France, 1-4 Sept 2008, 13 p.

Bonnet B., Marty A., Demante M.-J., 2005. Hydraulique et sécurisation des systèmes pastoraux au Sahel, appui à la gestion locale, IRAM - Institut de recherches et d'applications des méthodes de développement, 28 p. www. iram-fr.org/ouverturepdf.php?file=154.pdf

CILSS, 2018. Cadre Harmonisé d'analyse et d'identification des zones à risque et des populations en insécurité alimentaire et nutritionnelle au Sahel et en Afrique de l'Ouest $(\mathrm{CH})$-Analyse régionale de la situation de l'insécurité alimentaire et nutritionnelle aiguë - Situation courante (marsmai 2018) et projetée (Juin-Août 2018), CILSS, Ouagadougou, Burkina Faso

Fafchamps M., Gavian S., 1998. The Determinants of Livestock Prices in Niger, J. Afr. Econ., 6 (2): 255-295

FAO-PAM, 2017. Sécurité Alimentaire et Implications Humanitaires en Afrique de l'Ouest et au Sahel, Aout-Sept. 2017, 84, 3 p. www.fao. org/fileadmin/user_upload/emergencies/docs/Note\%20Conjointe_ Ao\%C3\%BBt $\% 20-\% 20$ Septembre\%202017_FAO_PAM_fr_vf.pdf

Fillol E., 2010. HydroGenerator (v1.1) Guide de I'utilisateur, Action contre la Faim International, Ecquevilly, France,15 p.

Fillol E., 2013. BioGenerator (v2.1) Guide de l'utilisateur, Action contre la Faim International, Ecquevilly, France, $12 \mathrm{p}$.

Fillol E., Métais T., Gomez A., 2008. Estimation de la quantité de biomasse sur la zone Sahélienne Mali-Niger par télédétection pour I'aide à la gestion de I'activité pastorale, Space Application Congress, Toulouse, France, 22-25 Avril 2008

Ford E., 2013. Learning the lessons? Assessing the response to the 2012 food crisis in the Sahel to build resilience for the future, Briefing paper, Oxfam, Oxford, Grande Bretagne p.16

FSNWG, 2017. Compte rendu de la réunion du groupe régional Sécurité Alimentaire et Nutrition 31 aout 2017, Dakar, Sénégal www.humanitarianresponse.info/ sites/www.humanitarianresponse.info/files/documents/files/ cr_fsnwg_31aout_vf.pdf

Haas E., 2009. Time series analysis of optical remote sensing data for the mapping of temporary surface water bodies in sub-Saharan western Africa, J. Hydrol., 12 p.

Haas E., 2010. Temporary water bodies as ecological indicators in West African drylands, Thèse Doct., Université Catholique de Louvain, Belgique, $226 \mathrm{p}$.

Ham F., 2013. Analyse de production de biomasse - Saison des pluies 2013, Document technique, Action contre la Faim International, Ecquevilly, France, $18 \mathrm{p}$.

Ham F., Fillol E., 2012. Pastoral Surveillance System and Feed Inventory in Sahel, Conducting National Feed Assessments, 83-94FAO, Rome, Italie

Ham F., Métais T., Hoorelbeke P., Fillol E., Gomez A., Crahay P., 2011. One horn of the cow, an innovative GIS-based surveillance and early warning system in pastoral areas of Sahel. In: Risk Returns: 127-131 UN Intern. Strat. Disaster Reduc. Sec. (UNISDR)

Ickowicz A., Ancey V., Leclerc G., Toure I., Damman G., Darly S., 2005. Rapport final du ProgrammeLEAD "Pastoralisme et Environnement au Sahel » (PESah), $108 \mathrm{p}$.

Illiassou I., 2017. Surveillance pastorale Niger : 2015-2017, Rapport d'activités du projet Surveillance Pastorale financé par la Fondation Albert II de Monaco, Novembre 2017, 14p. http://sigsahel.info/wp-content/ uploads/2017/12/Rapport_Activit\%C3\%A9s-SP_2015-2017.pdf

Jacobs R., Piccard I., Bydekerke L., Gontier E., 2006. Dry Matter Productivity. VGT4Africa User Manual (first ed), Office for Official Publication of the European Communities, Luxembourg, 271 p.

Marty A., 2000. Les approches de la gestion des pâturages et les projets de développement : quelles perspectives ? Note de l'atelier régional portant sur la Zone Sahélienne Ouest Africaine, Niamey, Niger, 2 - 6 Oct. 2000, $17 \mathrm{p}$.

Marty A., Bonnet B., Guibert B., Swift J., 2006. La mobilité pastorale et sa viabilité. Entre atouts et défis. Note thématique IRAM, 3 Juil. 2006, 4 p.

Métais T., 2007. Suivi des pâturages au Sahel. Spot mag. 42: 8-10

Milleville P., 1992. Conditions sahéliennes et déplacements des troupeaux bovins (Oudalan, Burkina Faso). ORSTOM, Paris, France, 539-553. (Didactiques). 
OCHA, 2018. Sahel: Overview of Humanitarian Needs and Requirements, https://reliefweb.int/sites/reliefweb.int/files/resources/Sahel\%20HNRO $\% 20$ 2018.pdf

Orenstein A., 2017a. Where the grass meets the sky, FOSS4G, Boston, EtatsUnis, 14-19 Aout 2017, vimeo.com/246731114

Orenstein A., 2017b. Réunion sur la Soudure Pastorale, FSN WG, Food Security and Nutrition Working Group, Dakar, Sénégal 2 Nov. 2017, www. humanitarianresponse.info/sites/www.humanitarianresponse.info/files/ documents/files/cr_fsnwg_31aout_vf.pdf

Ouedraogo I., 1994. Systèmes pastoraux et gestion des parcours dans la région de Banh au Nord-Yatenga, Séminaire régional sur la promotion de systèmes agricoles durables dans les pays d'Afrique soudano-sahélienne, Dakar, Sénégal : 10-14 Janv. 1994

PESAH, 2004. Elaboration d'un Système d'Information Pastoralisme et Environnement au Sahel (SIPES), Pastoralisme et Environnement au Sahel (PESAH), Compte-Rendu de l'Atelier régional de restitution du programme LEAD, Dakar, Sénégal, 1-2 Juil. 2004

Peyton N., 2017. Sahel herders facing harshest dry season in years, aid agency warns. Thomson Reuters Foundation, 27 Oct. 2017, www.reuters.com/ article/us-africa-herders/sahel-herders-facing-harshest-dry-season-in-yearsaid-agency-warns-idUSKBN1CW1ZF

Paridaens A.-M., 2017. Rapport d'évaluation Projet Surveillance Pastorale 2014-2017, Document technique interne, ACF, https://sigsahel.info/wpcontent/uploads/2018/01/Rapport_EvaluationFinale_ACFWA_DK00362. pdf

PRAPS-BF, 2017. Élaboration des outils de collecte et de suivi de données sur le pastoralisme au Burkina Faso, Rapport Final, Septembre 2017, Projet Régional d'Appui au Pastoralisme au Sahel, Burkina Faso www.praps.bf/ sites/default/files/elaboration_outil_collecte.pdf
RFI, 2017. Sahel : la saison pastorale pour I'an prochain risque d'être laborieuse, 30 Nov. 2017, www.rfi.fr/afrique/20171130-sahel-saison-pastoralean-prochain-risque-etre-laborieuse

Roucaute D., 2012. Le Sahel menacé par une crise alimentaire prévue depuis octobre, Le Monde, 10 Fév. 2012

Rouse L., 2018. Early Warning, late response to Senegal's food crisis, New Humanit., 18 Oct. 2018, www.thenewhumanitarian.org/special-report/2018/10/18/early-warnings-climate-change-senegal-food-crisis

RPCA, 2017. Avis sur les perspectives agricoles et alimentaires 2017-18 au Sahel et en Afrique de l'Ouest, Concertation technique du Dispositif Régional de Prévention et de Gestion des Crises Alimentaires (PREGEC), Bamako, Mali, 27-29 Nov. 2017

SAP Mali, 2017. Note sur le suivi des pâturages, Système d'alerte précoce de la République du Mali (SAP), 3 p., https://fscluster.org/sites/default/files/ documents/mali_-_analyse_biomasse_fin_saison_2017-2018_vf.pdf

Toutain B., Lhoste P., 1978. Estimation test of the coefficient of herbaceaous biomass utilization by cattle in a Sahelian area, Rev. Elev. Med. Vet. Pays trop., 31 (1) : 95-101, doi : 10.19182/remvt.8186

Toutain B., Ickowicz A., Dutilly-Diane C., Reid R.-S. Tamsir Diop A., Teneja V.-K., Gibon A., et al., 2010. Impact of extensive livestock systems on terrestrial ecosystems, in Livestock in a changing landscape. In: vol. 1, Ed. Steinfeld H., Mooney H.A., Schneider F., Neville L.E., 165-196, Islandpress, Washington, Etats-Unis,

Touré I., Ickowicz A., Wane A., Garba I., Gerber P., 2012. Système d'information sur le pastoralisme au Sahel : Atlas des évolutions des systèmes pastoraux au Sahel, 1970-2012. FAO, Rome, Italie, Cirad, Paris, France. https://umr-selmet.cirad.fr/content/download/3919/28448/version/1/file/ Atlas_SIPSA_2012-1.pdf

\section{Summary}

Fillol E., Ham F., Orenstein A. Information and modeling system of pastoral vulnerability for crisis management and prevention in the Sahel

Pastoral livestock herding in the Sahel is extremely sensitive to climatic irregularities and particularly to the quality of the rainy season that conditions the regrowth of pastures and the filling of water points. Nomadic and transhumant herders adapt their movement strategies to the state and availability of these resources. Tools for monitoring food security in these pastoral territories must therefore integrate, in addition to spatialized biomass data, information on pastoral movements and the factors influencing them. To support local warning systems in the Sahel, the non-governmental organization Action against Hunger proposes a pastoral vulnerability model based on real-time satellite data and on knowledge of pastoral practices. This model is used to process data into an early warning system by identifying pastoral resource-deficient areas several months before a potentially problematic tide-over season. The combination of this data with concomitant field information collected by sentinel relays enables monitoring pastoral conditions, thus contributing to infrastructure development or the deployment of humanitarian aid.

Keywords: livestock, pastoralism, biomass, pastures, water resources, early warning systems, vulnerability, Sahel

\section{Resumen}

Fillol E., Ham F., Orenstein A. Sistema de información y de modelización de la vulnerabilidad pastoral para la gestión y la prevención de crisis en el Sahel

La cría pastoral en el Sahel es extremadamente dependiente de las irregularidades climáticas y particularmente de la calidad de la estación lluviosa, que condiciona el rebrote de pastos y el Ilenado de las fuentes de agua. Los criadores nómadas y trashumantes adaptan sus estrategias de desplazamiento al estado y a la disponibilidad de estos recursos. Los instrumentos de seguimiento de la seguridad alimenticia de estos territorios pastorales deben así integrar, además de los datos espaciales de la biomasa, informaciones sobre estos movimientos pastorales y sobre los factores que los condicionan. Para acompañar los sistemas de alerta locales en el Sahel, la organización a fin no lucrativo Acción contra el Hambre propone un modelo de vulnerabilidad pastoral basado en los datos satelitales adquiridos en tiempo real y sobre el conocimiento de las prácticas pastorales. Este modelo permite alimentar informaciones en un sistema de alerta precoz, identificando las zonas de interés pastoral en déficit de recursos, varios meses antes del periodo de enlace potencialmente problemático. La confrontación con la información concomitante recopilada en el campo mediante relés centinela permite monitorear la situación pastoral, contribuyendo al desarrollo de infraestructuras o al despliegue de ayuda humanitaria.

Palabras clave: ganado, pastoralismo, biomasa, pastizales, recursos hídricos, sistemas de alerta rápida, vulnerabilidad, Sahel 
